University of Wollongong

Research Online

Faculty of Engineering and Information

Faculty of Engineering and Information

Sciences - Papers: Part A

Sciences

$1-1-2014$

Mean curvature flow of entire graphs in a half-space with a free boundary

V.-M Wheeler

University of Wollongong, vwheeler@uow.edu.au

Follow this and additional works at: https://ro.uow.edu.au/eispapers

Part of the Engineering Commons, and the Science and Technology Studies Commons

Research Online is the open access institutional repository for the University of Wollongong. For further information contact the UOW Library: research-pubs@uow.edu.au 


\title{
Mean curvature flow of entire graphs in a half-space with a free boundary
}

\author{
Abstract \\ We study the mean curvature flow of graphs with prescribed contact angle on a fixed, smooth hyperplane \\ in Euclidean space. We obtain long time existence and convergence to a self similar solution of the mean \\ curvature flow orthogonal to the fixed hyperplane. \\ Keywords \\ boundary, curvature, flow, entire, graphs, half, space, free, mean \\ Disciplines \\ Engineering | Science and Technology Studies \\ Publication Details \\ Vulcanov, V. (2014). Mean curvature flow of entire graphs in a half-space with a free boundary. Journal fur \\ die Reine und Angewandte Mathematik, 2014 (690), 115-131.
}

This journal article is available at Research Online: https://ro.uow.edu.au/eispapers/2262 


\title{
MEAN CURVATURE FLOW OF ENTIRE GRAPHS IN A HALF-SPACE WITH A FREE BOUNDARY
}

\author{
VALENTINA MIRA WHEELER
}

\begin{abstract}
We study the mean curvature flow of graphs with prescribed contact angle on a fixed, smooth hyperplane in Euclidean space. We obtain long time existence and convergence to a self similar solution of mean curvature flow orthogonal to the fixed hyperplane.
\end{abstract}

\section{INTRODUCTION}

There has been much work on the mean curvature flow problem for immersions and graphs with or without boundary conditions. The study of entire graphs over $\mathbb{R}^{n}$ by Ecker-Huisken $[4,5]$ provides a detailed exposition including a long time existence theorem for Lipschitz initial data. The non-parametric mean curvature flow of graphs with either a ninety degree contact angle or Dirichlet boundary condition on cylindrical domains has been studied by Huisken [9] and provides a long time existence and convergence to minimal surfaces theorem. In this direction we also mention the work of Altschuler-Wu [1] which allows arbitrary contact angle at the boundary for graphs over $\mathbb{R}^{2}$ and the generalisation to arbitrary intrinsic dimension by Guan [6]. A natural next step in this line of research is to study the mean curvature flow of graphs with a free boundary on a fixed hypersurface in $\mathbb{R}^{n+1}$. This began with a series of results on the mean curvature flow of immersions with free boundary, where a restriction on the angle of contact with a fixed hypersurface in Euclidean space is imposed. In [13] Stahl proves that the immersions either exist for all time or develop a curvature singularity. Also in the special case of convex initial hypersurfaces and umbilic, convex contact hypersurfaces he proves finite time blow up of curvature with the rescaled solution asymptotic to a hemisphere. Buckland [2], using a localized reflection in a neighbourhood of the boundary, proves the analogous monotonicity formula for mean curvature flow with a free boundary. He also provides us (again in the case of umbilic, convex contact hypersurfaces) with a classification of Type I singularities on the boundary. Regularity theory for this problem has been developed by Koeller [10] using the reflection construction of Buckland and the local boundary estimates of Stahl. He obtains the analogous results of regularity theory for compact mean curvature flow, see [3], in the boundary case.

In this paper we consider the mean curvature flow of graphs with a free boundary on a hyperplane in $\mathbb{R}^{n+1}$. In particular, suppose $\Sigma$ is an $n$-hyperplane in $\mathbb{R}^{n+1}$ with $n \geq 2$ defined by

$$
\left\langle\nu_{\Sigma}, e_{n+1}\right\rangle=0 .
$$

2000 Mathematics Subject Classification. 53C44 and 58J35.

Key words and phrases. mean curvature flow, free boundary conditions, geometric analysis. 
Define the two half spaces generated by $\Sigma$ in $\mathbb{R}^{n+1}$ as $\mathbb{R}_{+}^{n+1}$ and $\mathbb{R}_{-}^{n+1}$. Suppose $M^{n}$ is a smooth, orientable $n$-dimensional Riemaniann manifold with smooth boundary $\partial M^{n}$ and set $M_{0}:=F_{0}\left(M^{n}\right) \subset \mathbb{R}_{+}^{n+1}$ where $F_{0}: M^{n} \rightarrow \mathbb{R}^{n+1}$ is a smooth embedding satisfying

$$
\begin{aligned}
& \partial M_{0} \equiv F_{0}\left(\partial M^{n}\right)=M_{0} \cap \Sigma, \\
& \left\langle\nu_{M_{0}}, \nu_{\Sigma} \circ F_{0}\right\rangle(p)=0 \text { for all } p \in \partial M^{n},
\end{aligned}
$$

where we have denoted by $\nu_{M_{0}}$ the outer unit normal vector field on $M_{0}$ and we have taken the convention that $\nu_{\Sigma}$ points away from $M_{0}$.

Let $F_{t}=F(\cdot, t): M^{n} \rightarrow \mathbb{R}^{n+1}, t \in[0, T)$ be a one-parameter family of smooth embeddings. The family of hypersurfaces $\left(M_{t}\right)_{t \in[0, T)}$, where $M_{t}=F_{t}\left(M^{n}\right)$, is said to be evolving by mean curvature flow with a free Neumann boundary condition on $\Sigma$ if

$$
\begin{aligned}
\frac{\partial F}{\partial t}(p, t) & =-H(p, t) \nu_{M_{t}}, & & \text { for all }(p, t) \in M^{n} \times[0, T), \\
F(\cdot, 0) & =F_{0}, & & \\
F(p, t) & \subset \Sigma, & & \text { for all }(p, t) \in \partial M^{n} \times[0, T), \\
\left\langle\nu_{M_{t}}, \nu_{\Sigma} \circ F\right\rangle(p, t) & =0, & & \text { for all }(p, t) \in \partial_{N} M^{n} \times[0, T),
\end{aligned}
$$

where we have denoted by $\nu_{M_{t}}$ the unit normal to $M_{t}$ and by $H$ the mean curvature of $M_{t}$. We assume that the initial hypersurface $M_{0}$ is a graph in all points, that is

$$
\left\langle\nu_{M_{0}}, e_{n+1}\right\rangle \geq C_{s}>0
$$

where $C_{s}$ is a constant. Our goal is to preserve this condition for all times and use this to prove long time existence of the problem (2).

In [4], the maximum principle is combined with the evolution equations of various quantities and uniform bounds on height, gradient, curvature and derivatives of curvature are obtained. These are used to prove long time existence and convergence results. We apply the same arguments in the case of the boundary problem (2). The main difficulties encountered are in treating the boundary terms which appear. We notice that in the particular case of a hyperplane for the contact hypersurface, we are able to control all the important quantities on the boundary, such as the height, gradient, curvature and first derivatives of curvature. This is done by exploiting the Neumann boundary condition and making an appropriate choice of coordinates. A natural application of the maximum principle and Hopf Lemma allows us to obtain results analogous to those in [4] for the boundary case. We use these to prove long time existence by applying standard parabolic theory. We also discuss the sharpness of the convergence results.

Theorem 1.1 (Long time existence). Let $\Sigma$ be a hyperplane in $\mathbb{R}^{n+1}$ with $n \geq 2$ and $\left\langle\nu_{\Sigma}, e_{n+1}\right\rangle=0$. Suppose $F_{t}$ satisfies (2) and $M_{0}$ satisfies (3). Then there exists a smooth solution of (2) for all times $t \geq 0$.

The following result classifies the asymptotic behaviour. For this purpose we define as in [4] the rescaled flow to be

$$
\tilde{F}(r)=\frac{1}{\sqrt{2 t+1}} F(t)
$$

where the new time variable $r$ is given by $r=\frac{1}{2} \log (2 t+1), 0 \leq r<\infty$. 
Theorem 1.2 (Convergence). Let $\Sigma$ be a hyperplane in $\mathbb{R}^{n+1}$ with $n \geq 2$ and $\left\langle\nu_{\Sigma}, e_{n+1}\right\rangle=0$. Suppose $F_{t}$ satisfies (2) and $M_{0}$ satisfies (3). If $|u(\cdot, 0)| \leq C_{u}$ for some global constant $C_{u}<\infty$ then the hypersurfaces $M_{t}$ converge as $t \rightarrow \infty$ to a hyperplane orthogonal to $\Sigma$. Otherwise, if $0 \in \Sigma$ and $M_{0}$ satisfies the initial condition

$$
\left\langle F, \nu_{M_{t}}\right\rangle^{2} \leq C_{f}\left(1+|F|^{2}\right)^{1-\delta}
$$

for some constants $C_{f}<\infty, \delta>0$, then the rescaled solution $\tilde{M}_{r}=\tilde{F}(\cdot, r)\left(M^{n}\right)$ converges for $r \rightarrow \infty$ to a limiting hypersurface $\tilde{M}_{\infty}$ satisfying the equation

$$
\tilde{F}^{\perp}=-\tilde{H} \tilde{\nu} .
$$

Remark. The condition $0 \in \Sigma$ is just a formality since it can be obtained for arbitrary $\Sigma$ by translation, and (2) is invariant under translations.

The paper is organized as follows. In Section 2 we briefly discuss some maximum principle and Hopf Lemma tools. We omit these proofs since they can be easily adapted to our setting from the existing literature. Section 3 is concerned with obtaining the uniform estimates used in the proof of long time existence and the convergence discussion. These estimates include a height, gradient, curvature, derivatives of curvature estimates and also a decay of curvature in time. In Section 4 we give the proof of the long time existence Theorem 1.1 followed in Section 5 by the discussion of the asymptotic behaviour and proof of Theorem 1.2. In Section 6 we show that the convergence result is optimal.

\section{MAXIMUM PRINCIPLES}

In this section we present a collection of maximum principles for the mean curvature flow of immersions with boundary. We follow the results of the compact case found in [3] and modify the conditions to suit our boundary problem. We omit the proofs and note that the necessary modifications for the free boundary case can be found in [14].

Theorem 2.1 (Weak maximum principle for mean curvature flow with boundaries). Let $\left(M_{t}\right)_{t \in\left(t_{0}, t_{1}\right)}$ be a solution of mean curvature flow consisting of hypersurfaces $M_{t}=F_{t}\left(M^{n}\right)$ where $F(\cdot, t)=F_{t}: M^{n} \rightarrow \mathbb{R}^{n+1}$ and $M^{n}$ has a smooth boundary $\partial M^{n}$. Suppose $h: \bigcup_{t \in\left[t_{0}, t_{1}\right)} M_{t} \times\{t\} \rightarrow \mathbb{R}$ is sufficiently smooth for $t>t_{0}$, continuous on $\bigcup_{t \in\left[t_{0}, t_{1}\right]} M_{t} \times\{t\}$ and satisfies an inequality of the form

$$
\left(\frac{d}{d t}-\Delta^{M_{t}}\right) h \leq a \cdot \nabla h
$$

where we have denoted by $\nabla$ the covariant derivative on $M_{t}$. Then

$$
\sup _{M_{t}} h \leq \max \left\{\sup _{M_{0}} h, \sup _{\partial M_{t}} h\right\}
$$

for all $t \in\left[t_{0}, t_{1}\right]$. For the vector $a: \bigcup_{t \in\left[t_{0}, t_{1}\right)} M_{t} \times\{t\} \rightarrow \mathbb{R}^{n+1}$ we only require that it is well-defined and bounded in a neighbourhood of all maxima of $h$. 
Following Huisken [8] we state a comparison principle for two solutions of mean curvature flow with free Neumann boundaries on a fixed hypersurface $\Sigma$. In the proof the only required modification is the one dealing with possible contact of the evolving hypersurfaces on the boundary.

Theorem 2.2 (Comparison principle for mean curvature flow with boundaries). Let $M_{1}$ and $M_{2}$ be two smooth solutions of mean curvature flow (2) for an arbitrary smooth hypersurface $\Sigma$ for time $0 \leq t \leq t_{1}$. If $\overline{M_{1}}$ and $\overline{M_{2}}$ are disjoint at time $t=0$ then they remain disjoint for the whole interval $0 \leq t \leq t_{1}$.

Finally we add a Hopf Lemma to our collection of maximum principles for hypersurfaces moving by mean curvature flow.

Lemma 2.3 (Hopf Lemma for mean curvature flow). Let $\left(M_{t}\right)_{t \in\left(t_{0}, t_{1}\right)}$ be a smooth solution of mean curvature flow consisting of hypersurfaces $M_{t}=F_{t}\left(M^{n}\right)$ where $F(\cdot, t)=F_{t}: M^{n} \rightarrow \mathbb{R}^{n+1}$ and $M^{n}$ has a smooth boundary $\partial M^{n}$. Suppose $h:$ $\bigcup_{t \in\left[t_{0}, t_{1}\right)} M_{t} \times\{t\} \rightarrow \mathbb{R}$ is sufficiently smooth for $t>t_{0}$, continuous on $\bigcup_{t \in\left[t_{0}, t_{1}\right]} M_{t} \times\{t\}$ and satisfies an inequality of the form (7). Take $p^{*} \in \partial M^{n}$ and a time $t^{*}$ such that at the point $X^{*}=F\left(p^{*}, t^{*}\right), h$ attains a first maximum. Then at this point we have

$$
\nabla_{\nu_{\partial M_{t^{*}}}} h\left(X^{*}\right)>0
$$

where $\nu_{\partial M_{t}}$ is the normal to the boundary $\partial M_{t}$. In case $X^{*}$ is a point where $h$ attains a first minimum then the sign of the inequality changes.

Remark. The smoothness of $M_{t}$ together with the assumption that we work only at a first maximum or minimum allows us to obtain the above result in space time domains with corners, as in [12].

\section{UNIFORM BOUNDS}

In this section we present how one can obtain the uniform estimates and decay behaviour required for the long time existence and convergence results.

Following [4] we define the inverse of the gradient of the associated graph function to $M_{t}$ as $s: M_{t} \rightarrow \mathbb{R}$ and the height function $u: M_{t} \rightarrow \mathbb{R}$ as

$$
s(F)=\left\langle\nu_{M_{t}}, e_{n+1}\right\rangle \text {, and } u(F)=\left\langle F, e_{n+1}\right\rangle .
$$

The following lemma contains the evolution equations of various quantities along the mean curvature flow. Detailed proofs can be found in $[4,7]$.

Lemma 3.1 (Evolution equations). Let $\left(M_{t}\right)_{t \in[0, T)}$ be a solution of mean curvature flow consisting of hypersurfaces $M_{t}=F_{t}\left(M^{n}\right)$ where $F(\cdot, t)=F_{t}: M^{n} \rightarrow \mathbb{R}^{n+1}$, with or without a smooth boundary. Then one has the following evolution equations

$$
\begin{aligned}
\left(\frac{d}{d t}-\Delta^{M_{t}}\right) u & =0, \\
\left(\frac{d}{d t}-\Delta^{M_{t}}\right) s & =|A|^{2} s, \\
\left(\frac{d}{d t}-\Delta^{M_{t}}\right)|A|^{2} & =-2|\nabla A|^{2}+|A|^{4}, \\
\left(\frac{d}{d t}-\Delta^{M_{t}}\right)|\nabla A|^{2} & =-2\left|\nabla^{2} A\right|^{2}+C|A|^{2}|\nabla A|^{2},
\end{aligned}
$$


where $C$ is an absolute constant and we denoted by $|A|^{2}$ the square of the induced norm of the second fundamental form of $M_{t}$.

Here and henceforth we denote by $h_{i j}$ and $h_{i j}^{\Sigma}$ the components of the second fundamental forms of $M_{t}$ and $\Sigma$ respectively. Due to the Neumann boundary condition, in an orthonormal frame on the boundary $\partial M_{t} \subset \Sigma$, we may choose the $n$-th index to correspond to $\nu_{\Sigma}$ for the second fundamental form of $M_{t}$ and to $\nu_{M_{t}}$ for the second fundamental form of $\Sigma$.

To treat the boundary terms in the following we use a special choice of coordinates.

Lemma 3.2 (Fermi coordinates on the boundary). Let $F_{t}$ satisfy (2) for a general hypersurface $\Sigma \subset \mathbb{R}^{n+1}$. Given a point $X=F(p, t) \in \partial M_{t} \subset \Sigma$, we can introduce local coordinates around $p$ in a neighbourhood $\mathcal{U}_{p} \subset M^{n}$ such that the vector fields $\tau_{i}:=\frac{\partial F}{\partial p_{i}}(p, t), i=1, \ldots, n-1, \tau_{n}:=\frac{\partial F}{\partial p_{n}}(p, t)=\nu_{\Sigma}(X)=\nu_{\partial M_{t}}(X)$ and $\tau_{n+1}=\nu_{M_{t}}(X)$ are orthonormal at $X$. Here we have denoted by $\nu_{\partial M_{t}}$ the outer unit normal to the boundary $\partial M_{t}$. In these coordinates we have $\left\langle\tau_{n}, \tau_{i}\right\rangle(F(p, t))=0$ and $\left\langle\nabla_{\tau_{n}} \tau_{k}, \tau_{s}\right\rangle(F(p, t))=h_{k s}^{\Sigma}(F(p, t))$ for all $p \in \mathcal{U}_{p} \cap \partial M^{n}$ and for each $i, k, s=$ $1, \ldots, n-1$.

Proof. The choice of local coordinates is done in the following way. We have that $\partial M_{t}=F_{t}\left(\partial M^{n}\right)$ and let $X=F(p, t), p \in \partial M^{n}$ be a boundary point. Choose normal local coordinates $p_{1}, \ldots, p_{n-1}$ in a neighbourhood $\mathcal{W}_{p} \subset \partial M^{n}$ of the point $p$ on the boundary such that $\tau_{i}:=\frac{\partial F}{\partial p_{i}}$ form a basis of $T_{p} \partial M_{t}$ for all points in $\mathcal{W}_{p}$. Note that only at $X=F(p, t)$ can we choose it to be orthonormal.

Let $\gamma\left(p_{n}\right)$ be a geodesic leaving from $\left(p_{1}, \ldots, p_{n-1}\right)$ in the direction orthogonal to $\partial M^{n}$ and parametrised by arc length. The coordinates $p_{1}, \ldots, p_{n}$ are called the Fermi coordinates at $p$ in the neighbounhood $\mathcal{U}_{p} \subset M^{n}$ containing $\mathcal{W}_{p} \subset \partial M^{n}$. In these coordinates on the boundary neighbourhood $\mathcal{W}_{p}$ the metric is given by a block matrix where $g_{i n}=0$ for each $i=1, \ldots, n-1$. One can also see that, due to the Neumann boundary condition, $\nu_{\Sigma}=\frac{\partial F}{\partial p_{n}}$ at every point in $\mathcal{W}_{p}$ on the boundary. In these coordinates we have

$$
\begin{aligned}
\left\langle\nabla_{\tau_{n}} \tau_{k}, \tau_{s}\right\rangle(F(p, t)) & =\left\langle\frac{\partial^{2} F}{\partial p_{n} \partial p_{k}}, \frac{\partial F}{\partial p_{s}}\right\rangle(p, t)=\left\langle\frac{\partial^{2} F}{\partial p_{k} \partial p_{n}}, \frac{\partial F}{\partial p_{s}}\right\rangle(p, t) \\
& =\left\langle\nabla_{\tau_{k}} \tau_{n}, \tau_{s}\right\rangle(F(p, t))=\left\langle\nabla_{\tau_{k}} \nu_{\Sigma}, \tau_{s}\right\rangle(F(p, t)) \\
& =h_{k s}^{\Sigma}(F(p, t)),
\end{aligned}
$$

for any $k, s=1, \ldots, n-1$.

This relates the formalism of orthonormal moving frames and local coordinate systems. Note that if $\Sigma$ is a hyperplane this implies $\left\langle\nabla_{\tau_{n}} \tau_{k}, \tau_{s}\right\rangle=h_{k s}^{\Sigma}=0$ for any $s, k=1, \ldots, n-1$.

The following proposition from [13] relates the curvatures of an umbilic hypersurface $\Sigma$ and those of the moving hypersurfaces $M_{t}$. If $\Sigma$ is umbilic then there exists a function $\alpha: \Sigma \rightarrow \mathbb{R}$ such that $h_{i j}^{\Sigma}(X)=\alpha g_{i j}^{\Sigma}(X)$ for any point $X \in \Sigma$.

Proposition 3.3 (Stahl [13]). Let $\Sigma \subset \mathbb{R}^{n+1}$ be umbilic and $F_{t}$ satisfy (2). Suppose $X \in \partial M_{t} \subset \Sigma$ is a boundary point and choose local coordinates around $X$ as in 
Lemma 3.2. Then the following relations hold:

$$
\begin{aligned}
h_{i n} & =-h_{i n}^{\Sigma}=0, \\
\nabla_{n} h_{i j} & =\alpha\left(-h_{i j}+h_{n n} \delta_{i j}\right), \\
\nabla_{n} h_{n n} & =\alpha\left(2 H-n h_{n n}\right), \\
\nabla_{n} H & =\alpha H,
\end{aligned}
$$

for any $i, j=1, \ldots, n-1$ and where $\delta_{i j}$ are the Krönecker symbols.

Notice that, since $\alpha \equiv 0$ when $\Sigma$ is a hyperplane, the above proposition implies that all the quantities vanish on the boundary. In the following we prove that some of the second derivatives of curvatures also vanish.

Proposition 3.4 (Boundary derivatives of curvature). Let $\Sigma$ be a hyperplane and $F_{t}$ satisfy (2). Suppose $X \in \partial M_{t} \subset \Sigma$ is a boundary point and choose local coordinates around $X$ as in Lemma 3.2. Then the following relations hold:

$$
\begin{aligned}
\nabla_{k} \nabla_{n} h_{i j} & =0 \\
\nabla_{k} \nabla_{n} h_{n n} & =0,
\end{aligned}
$$

for any $i, j, k=1, \ldots, n-1$.

Proof. For $\Sigma$ a hyperplane, Proposition 3.3 implies

$$
\begin{aligned}
\nabla_{n} h_{i j} & =0, \text { and } \\
\nabla_{n} h_{n n} & =0,
\end{aligned}
$$

everywhere on $\partial M_{t}$. We can therefore differentiate these relations in any directions tangential to $\partial M_{t}$ to obtain

$$
\begin{aligned}
\nabla_{k} \nabla_{n} h_{i j}= & \mathrm{D}_{\tau_{k}}\left(\nabla_{\tau_{n}} A\left(\tau_{i}, \tau_{j}\right)\right)-\sum_{l=1}^{n} \nabla_{l} h_{i j}\left\langle\nabla_{\tau_{k}} \tau_{n}, \tau_{l}\right\rangle \\
& -2 \sum_{l=1}^{n} \nabla_{n} h_{i l}\left\langle\nabla_{\tau_{k}} \tau_{j}, \tau_{l}\right\rangle \\
= & -\sum_{l=1}^{n} \nabla_{l} h_{i j}\left\langle\nabla_{\tau_{k}} \tau_{n}, \tau_{l}\right\rangle-2 \sum_{l=1}^{n} \nabla_{n} h_{i l}\left\langle\nabla_{\tau_{k}} \tau_{j}, \tau_{l}\right\rangle=0, \\
\nabla_{k} \nabla_{n} h_{n n}= & \mathrm{D}_{\tau_{k}}\left(\nabla_{\tau_{n}} A\left(\tau_{n}, \tau_{n}\right)\right)-3 \sum_{l=1}^{n} \nabla_{l} h_{n n}\left\langle\nabla_{\tau_{k}} \tau_{n}, \tau_{l}\right\rangle \\
= & -3 \sum_{l=1}^{n} \nabla_{l} h_{n n}\left\langle\nabla_{\tau_{k}} \tau_{n}, \tau_{l}\right\rangle=0,
\end{aligned}
$$

for any $k=1, \ldots, n-1$, where we have used the Codazzi equations in the first equalities. Here we have also denoted by $\mathrm{D}$ the covariant derivative in $\mathbb{R}^{n+1}$. To see that the first order terms vanish we used the fact that $\Sigma$ is a hyperplane for which $\left\langle\nabla_{\tau_{k}} \tau_{n}, \tau_{l}\right\rangle=-\left\langle\nabla_{\tau_{k}} \tau_{l}, \tau_{n}\right\rangle=h_{k l}^{\Sigma}=0$ and $\left\langle\nabla_{\tau_{k}} \tau_{n}, \tau_{n}\right\rangle=\left\langle\mathrm{D}_{\tau_{k}} \nu_{\Sigma}, \nu_{\Sigma}\right\rangle=0$ for a choice of coordinates as in Lemma 3.2 and for any $k, l=1, \ldots, n-1$ and also applied Proposition 3.3.

We now turn our attention to height bounds. 
Proposition 3.5 (Uniform height bounds). Let $\Sigma$ be a hyperplane in $\mathbb{R}^{n+1}$ with $n \geq 2$ and $\left\langle\nu_{\Sigma}, e_{n+1}\right\rangle=0$. Let $F_{t}$ satisfy (2) with $F_{0}$ satisfying the initial graph condition (3) everywhere on $M_{0}$. Then

$$
\sup _{M_{t}}|u| \leq \sup _{M_{0}}|u|
$$

for all times $t \in[0, T)$.

Proof. From the parabolic evolution of the height function (8) we obtain that the square of the height function also satisfies

$$
\left(\frac{d}{d t}-\Delta^{M_{t}}\right) u^{2}=-2|\nabla u|^{2} \leq 0 .
$$

The maximum principle then implies

$$
\sup _{M_{t}} u^{2} \leq \max \left\{\sup _{M_{0}} u^{2}, \max _{\partial M_{t}} u^{2}\right\} .
$$

To exclude a maximum of the square of the height function on the Neumann boundary we will make use of (1). Suppose there exists a point $X^{*}=X^{*}\left(p^{*}, t^{*}\right) \in \partial M_{t^{*}}$ such that the function $u^{2}$ attains a boundary maximum at $X^{*}$. Then by the Hopf Lemma, using the parabolic evolution of $u^{2}$, we obtain a sign on the derivative of the height function in any direction normal to the boundary. In particular,

$$
0<\left\langle\nabla u^{2}, \nu_{\partial M_{t}}\right\rangle\left(X^{*}\right)=2 u\left\langle\nabla u, \nu_{\Sigma}\right\rangle\left(X^{*}\right)
$$

where we have chosen $\nu_{\partial M_{t}}=\nu_{\Sigma}$. Note that the boundary condition ensures that we are always allowed to do this. With a choice of orthonormal frame $\left\{\tau_{i}\right\}_{i=1, n}$ of $T M_{t}$ such that $\left.\tau_{i}\right|_{\partial M_{t}} \in T \partial M_{t}$ for all $i=1, \ldots, n-1$ and $\left.\tau_{n}\right|_{\partial M_{t}}=\nu_{\Sigma}$ we have at $X^{*}$

$$
\left\langle\nabla u, \nu_{\Sigma}\right\rangle=\sum_{i=1}^{n}\left\langle\tau_{i}, e_{n+1}\right\rangle\left\langle\tau_{i}, \nu_{\Sigma}\right\rangle=\left\langle\nu_{\Sigma}, e_{n+1}\right\rangle=0
$$

from (1). This contradicts (13) and thus there is no maximum of the height squared on the Neumann boundary at any time. In view of (12) we are finished.

Observe that $\sqrt{1+|D w|^{2}}=\frac{1}{s}$ up to tangential diffeomorphisms, where we have denoted by $w$ the associated graph function of $M_{t}$. So bounding $s$ from below is equivalent to bounding the gradient of the associated graph function from above. With this in mind, we present the following uniform gradient estimate.

Proposition 3.6 (Uniform gradient bound). Let $\Sigma$ be a hyperplane in $\mathbb{R}^{n+1}$ with $n \geq 2$ and $\left\langle\nu_{\Sigma}, e_{n+1}\right\rangle=0$. Let $F_{t}$ satisfy (2) with $F_{0}$ satisfying the initial graph condition (3) everywhere on $M_{0}$. Then

$$
\inf _{M_{t}} s \geq \inf _{M_{0}} s,
$$

for all times $t \in[0, T)$.

Proof. The parabolic evolution of $s,(9)$ and the initial positivity (3) imply

$$
\inf _{M_{t}} s \geq \min \left\{\inf _{M_{0}} s, \inf _{\partial M_{t}} s\right\} .
$$


Suppose that there exists a point $X^{*}=F\left(p^{*}, t^{*}\right) \in \partial M_{t^{*}}$ such that $s$ attains a minimum value at $X^{*}$. Then by applying the Hopf Lemma with a choice of orthonormal frame as in Lemma 3.2 we have at $X^{*}$

$$
\begin{aligned}
0>\nabla_{\nu_{\Sigma}} s & =\nabla_{\nu_{\Sigma}}\left\langle\nu_{M_{t}}, e_{n+1}\right\rangle=\sum_{i=1}^{n-1} h_{i n}\left\langle\tau_{i}, e_{n+1}\right\rangle+h_{n n}\left\langle\nu_{\Sigma}, e_{n+1}\right\rangle \\
& =\sum_{i=1}^{n-1} h_{i n}\left\langle\tau_{i}, e_{n+1}\right\rangle,
\end{aligned}
$$

where we have used the definition of the second fundamental form and, in the last equality, the fact that $\Sigma$ is a hyperplane with $e_{n+1} \in T \Sigma$. Using Proposition 3.3 we have for $\Sigma$ hyperplane

$$
h_{i n}=-h_{i n}^{\Sigma}=0,
$$

for all $i=1, \ldots, n-1$. This transforms the previous inequality to

$$
0>\nabla_{\nu_{\Sigma}} s=0
$$

and contradicts the existence of a first minimum of $s$ on the Neumann boundary.

Proposition 3.7 (Uniform curvature bounds). Let $\Sigma$ be a hyperplane in $\mathbb{R}^{n+1}$ with $n \geq 2$ and $\left\langle\nu_{\Sigma}, e_{n+1}\right\rangle=0$. Let $F_{t}$ satisfy $(2)$ with $F_{0}$ satisfying the initial graph condition (3) everywhere on $M_{0}$. Then

$$
\sup _{M_{t}} \frac{|A|^{2}}{s^{2}} \leq \sup _{M_{0}} \frac{|A|^{2}}{s^{2}}
$$

for all times $t \in[0, T)$.

Proof. The proof is based again on the application of the maximum principle. Following [4] we see from (9) and (10) that $\frac{|A|^{2}}{s^{2}}$ satisfies the parabolic evolution

$$
\left(\frac{d}{d t}-\Delta^{M_{t}}\right) \frac{|A|^{2}}{s^{2}} \leq 2 \frac{\nabla s}{s} \cdot \nabla \frac{|A|^{2}}{s^{2}} .
$$

Notice that $s$ is bounded uniformly away from zero by Proposition 3.6 and $|\nabla s|<\infty$ at any point where $|A|^{2}<\infty$. Therefore applying the maximum principle with the bounded vector field $a=2 \frac{\nabla s}{s}$, we see that so long as we exclude maxima of the above quantity on the boundary we obtain the desired uniform estimate. Consider the orthonormal frame constructed in Lemma 3.2 and suppose that there exists a point $X^{*}=F\left(p^{*}, t^{*}\right) \in \partial M_{t^{*}}$ such that $\frac{|A|^{2}}{s^{2}}$ attains a boundary maximum value at $X^{*}$. From the Hopf Lemma we have at $X^{*}$

$$
0<\nabla_{\nu_{\Sigma}} \frac{|A|^{2}}{s^{2}}=\frac{\nabla_{\nu_{\Sigma}}|A|^{2}}{s^{2}}-2 \frac{|A|^{2}}{s^{3}} \nabla_{\nu_{\Sigma}} s
$$

The definition of $|A|^{2}$ in the choice of orthonormal basis and Proposition $3.3 X^{*}$ implies:

$$
\begin{aligned}
\nabla_{\nu_{\Sigma}}|A|^{2} & =2 \sum_{i, k=1}^{n} h_{i k} \nabla_{n} h_{i k}+4 \sum_{i, k, s=1}^{n} h_{i s} h_{i k}\left\langle\nabla_{\tau_{n}} \tau_{k}, \tau_{s}\right\rangle \\
& =4 \sum_{i, k, s=1}^{n-1} h_{i s} h_{i k}\left\langle\nabla_{\tau_{n}} \tau_{k}, \tau_{s}\right\rangle
\end{aligned}
$$


Above we have used the Codazzi equations and the fact that $\Sigma$ is a hyperplane, for which $h_{i n}=-h_{i n}^{\Sigma}=0, \nabla_{i} h_{k n}=0$ and $\nabla_{n} h_{n n}=0$ for $i, k=1, \ldots, n-1$. Also due to the fact that $\Sigma$ is a hyperplane, with the special choice of local coordinates as in Lemma 3.2 at $X^{*}$ we can prove that $\left\langle\nabla_{\tau_{n}} \tau_{k}, \tau_{s}\right\rangle=h_{k s}^{\Sigma}=0$ for each $k, s=$ $1, \ldots, n-1$. We obtain

$$
\nabla_{\nu_{\Sigma}}|A|^{2}=0
$$

The second term in (14) also vanishes by a similar discussion as in the proof of the gradient bound. Replacing the two terms in (14) we conclude

$$
0<\nabla_{\nu_{\Sigma}} \frac{|A|^{2}}{s^{2}}=0
$$

Thus there does not exist a maximum of $\frac{|A|^{2}}{s^{2}}$ on the Neumann boundary and we obtain the desired bound.

Note that a similar bound on $H$ can be easily obtained from the above result by using the well known inequality $H^{2} \leq n|A|^{2}$.

Remark. Note that the above proposition together with the preservation of the initial graph condition (3) implies that we have a uniform bound on $|A|^{2}$.

We can also prove that the second fundamental form and its first derivatives decay in time.

Proposition 3.8 (Decay of curvature). Let $\Sigma$ be a hyperplane in $\mathbb{R}^{n+1}$ with $n \geq 2$ and $\left\langle\nu_{\Sigma}, e_{n+1}\right\rangle=0$. Let $F_{t}$ satisfy (2) with $F_{0}$ satisfying the initial graph condition (3) everywhere on $M_{0}$. Then we have

$$
\begin{aligned}
2 t|A|^{2} & \leq C_{s}^{-2}-1, \\
t^{2}|\nabla A|^{2} & \leq C_{d},
\end{aligned}
$$

for all times $t \in[0, T)$. Here $C_{d}=C_{d}\left(|A|^{2}, n\right)=C_{d}\left(M_{0}, n\right)$.

Proof. Following the idea of Ecker and Huisken [4], we compute the evolution on $M_{t}$ of $2 t \frac{|A|^{2}}{s^{2}}+\frac{1}{s^{2}}$ using the proof of Proposition 3.7 and exclude interior maxima. The boundary terms are treated in the same way.

For the first derivative one can establish an interior evolution depending on $|A|^{2}$ in the same way as [4] using Theorem 7.1 of [7] (cf. (9), (10), (11)):

$$
\left(\frac{d}{d t}-\Delta^{M_{t}}\right)\left(t^{2}|\nabla A|^{2}+k_{1} t|A|^{2}+k_{2} \frac{1}{s^{2}}\right) \leq 0
$$

where the constants $k_{1}$ and $k_{2}$ depend only on $C_{s}$ and $n$.

From the maximum principle it follows that if we can exclude boundary maxima of $t^{2}|\nabla A|^{2}+k_{1} t|A|^{2}+k_{2} \frac{1}{s^{2}}$ the second decay result is proved. On the boundary the directional derivatives in the direction normal to the boundary of the second and third terms vanish, as we have seen in Proposition 3.6 and Proposition 3.7. 
We proceed by computing the directional derivative of the first term using an orthonormal frame as in Lemma 3.2

$$
\begin{aligned}
\nabla_{\nu_{\Sigma}}|\nabla A|^{2} & =2 \sum_{i, j, k=1}^{n}\left(\nabla_{k} h_{i j}\right)\left(\nabla_{n} \nabla_{k} h_{i j}\right) \\
& +6 \sum_{i, j, k=1}^{n} \sum_{m=1}^{n}\left(\nabla_{k} h_{i j}\right)\left(\nabla_{m} h_{i j}\right)\left\langle\nabla_{\tau_{n}} \tau_{k}, \tau_{m}\right\rangle,
\end{aligned}
$$

where we have use Codazzi equations to shorten the notation. As above we make use of our choice of frame from Lemma 3.2 for which we have $\left\langle\nabla_{\tau_{n}} \tau_{k}, \tau_{m}\right\rangle=h_{k m}^{\Sigma}=0$ for any $k, m=1 \ldots, n-1$. Hence the above directional derivative simplifies to

$$
\nabla_{\nu_{\Sigma}}|\nabla A|^{2}=2 \sum_{i, j, k=1}^{n}\left(\nabla_{k} h_{i j}\right)\left(\nabla_{n} \nabla_{k} h_{i j}\right) .
$$

From Proposition 3.3 we have $\nabla_{n} h_{i j}=0$ for any $i, j=1, \ldots, n-1$, so in the triple sum above only some of the terms do not vanish

$$
\nabla_{\nu_{\Sigma}}|\nabla A|^{2}=2 \sum_{i, j, k=1}^{n-1}\left(\nabla_{k} h_{i j}\right)\left(\nabla_{n} \nabla_{k} h_{i j}\right)+6 \sum_{k=1}^{n-1}\left(\nabla_{k} h_{n n}\right)\left(\nabla_{n} \nabla_{k} h_{n n}\right) .
$$

We have again used the Codazzi equations. We wish to make use of the properties of the second derivatives of the second fundamental form, proved in Proposition 3.4. For this we interchange the covariant derivatives in the above second order terms. The first term becomes

$$
\begin{aligned}
\sum_{i, j, k=1}^{n-1}\left(\nabla_{k} h_{i j}\right)\left(\nabla_{n} \nabla_{k} h_{i j}\right)= & \sum_{i, j, k=1}^{n-1}\left(\nabla_{k} h_{i j}\right)\left(\nabla_{k} \nabla_{n} h_{i j}\right)+\sum_{i, j, k=1}^{n-1} \nabla_{k} h_{i j} \sum_{m, l=1}^{n} R_{l j k n} g^{l m} h_{i m} \\
& +\sum_{i, j, k=1}^{n-1} \nabla_{k} h_{i j} \sum_{m, l=1}^{n} R_{i l k n} g^{l m} h_{j m} \\
= & \sum_{i, j, k=1}^{n-1} \nabla_{k} h_{i j} \sum_{m, l=1}^{n}\left(h_{j n} h_{l k}-h_{n l} h_{j k}\right) g^{l m} h_{i m} \\
& +\sum_{i, j, k=1}^{n-1} \nabla_{k} h_{i j} \sum_{m, l=1}^{n}\left(h_{i k} h_{l n}-h_{i n} h_{l k}\right) g^{l m} h_{j m} \\
= & -\sum_{i, j, k=1}^{n-1} \nabla_{k} h_{i j} \sum_{m=1}^{n} h_{n n} h_{j k} g^{n m} h_{i m} \\
& +\sum_{i, j, k=1}^{n-1} \nabla_{k} h_{i j} \sum_{m=1}^{n} h_{n n} h_{i k} g^{n m} h_{j m} \\
= & 0,
\end{aligned}
$$

where we used the fact that we can express the components $R_{i j k l}$ of the Riemann tensor in terms of the second fundamental form, the choice of orthonormal frame and also Proposition 3.3. In the same way the second term can also be shown to 
vanish:

$$
\begin{aligned}
\sum_{k=1}^{n-1}\left(\nabla_{k} h_{n n}\right)\left(\nabla_{n} \nabla_{k} h_{n n}\right) & =2 \sum_{k=1}^{n-1} \nabla_{k} h_{n n} R_{n n k n} h_{n n} \\
& =2 \sum_{k=1}^{n-1} \nabla_{k} h_{n n}\left(h_{n n} h_{k n}-h_{n n} h_{k n}\right) h_{n n} \\
& =0 .
\end{aligned}
$$

We therefore conclude that

$$
\nabla_{\nu_{\Sigma}}|\nabla A|^{2}=0
$$

and as discussed before this provides us with the second decay estimate on $|\nabla A|^{2}$ by the use of Hopf Lemma.

Having obtained decay estimates in $t$ we are able to prove, as in [4], that an initial spatial decay behaviour is maintained. This will be used in Section 6 to show that the condition (5) is optimal.

Proposition 3.9 (Spatial decay). Let $\Sigma$ be a hyperplane in $\mathbb{R}^{n+1}$ with $n \geq 2$, $\left\langle\nu_{\Sigma}, e_{n+1}\right\rangle=0$ and $0 \in \Sigma$. Let $F_{t}$ satisfy $(2)$ with $F_{0}$ satisfying the initial graph condition (3) everywhere on $M_{0}$. If in addition $F_{0}$ satisfies

$$
\left|\nabla^{m} A\right|^{2} \leq \tilde{C}_{m}\left(1+|F|^{2}\right)^{-m-1} \text { for } m=0,1,
$$

then for all $t>0$

$$
\left|\nabla^{m} A\right|^{2} \leq C_{m}\left(1+\left(\sqrt{|F|^{2}+2 n t}-\sqrt{\beta t}\right)^{2}\right)^{-m-1}
$$

where $\beta=\beta\left(C_{s}\right)>0, C_{m}=C_{m}\left(n, m, C_{s}, \tilde{C}_{0}, \tilde{C}_{1}\right)$ and $m=0,1$.

Proof. Once again we follow Ecker-Huisken [4] and supply additional arguments required to deal with the boundary. To prove the inequality for $m=0$ one defines the function $g=\frac{|A|^{2}}{s^{2}} \mu+\frac{L}{s^{2}}$ with $\mu(F(p, t), t)=1+\left(\sqrt{|F|^{2}+2 n t}-\sqrt{\beta t}\right)^{2}$ where $L>0$ and $\beta>0$ are constants to be suitably chosen. We obtain the following evolution for $g$

$$
\left(\frac{d}{d t}-\Delta^{M_{t}}\right) g \leq b \cdot \nabla g-\frac{1}{\mu}(g-k),
$$

where $b=-2\left(-\frac{\nabla s}{s}+\frac{\nabla \mu}{\mu}\right)$ and $k=\sup _{M_{0}} g+9 \frac{L}{C_{s}^{2}}$. Thus for $(g-k)^{2}$ we have the evolution

$$
\left(\frac{d}{d t}-\Delta^{M_{t}}\right)(g-k)^{2} \leq b \cdot \nabla(g-k)^{2} .
$$

This implies that if we can exclude boundary maxima of $(g-k)^{2}$, we obtain the result for $m=0$.

To compute the directional derivative of $(g-k)^{2}$ we first choose an orthonormal frame as in Lemma 3.2. With this choice we compute

$$
\nabla_{\nu_{\Sigma}}(g-k)^{2}=2(g-k)\left(\frac{|A|^{2}}{s^{2}} \nabla_{\nu_{\Sigma}} \mu\right) .
$$


Above we used the fact that $\nabla_{\nu_{\Sigma}} s=\nabla_{\nu_{\Sigma}}|A|^{2}=0$ (cf. Propositions 3.6 and 3.7). The remaining term also vanishes as follows

$$
\begin{aligned}
\nabla_{\nu_{\Sigma}} \mu & =2\left(\sqrt{|F|^{2}+2 n t}-\sqrt{\beta t}\right) \frac{\left\langle\nabla_{\nu_{\Sigma}} F, F\right\rangle}{\sqrt{|F|^{2}+2 n t}} \\
& =2\left(\sqrt{|F|^{2}+2 n t}-\sqrt{\beta t}\right) \frac{\left\langle\nu_{\Sigma}, F\right\rangle}{\sqrt{|F|^{2}+2 n t}}=0,
\end{aligned}
$$

where we have used that $\left\langle\nu_{\Sigma}, F\right\rangle=0$ due to our assumption $0 \in \Sigma$. Thus by the Hopf Lemma we exclude boundary maxima of $(g-k)^{2}$ and obtain the estimate for $m=0$.

For $m=1$ we compute (cf. the proof of Proposition 3.8)

$$
\left(\frac{d}{d t}-\Delta^{M_{t}}\right)\left(|\nabla A|^{2} \mu^{2}+K|A|^{2} \mu+\frac{L}{s^{2}}\right) \leq 0,
$$

with a suitable choice of constants $L, K>0$. In the same way as in the above mentioned proposition the boundary directional derivative vanishes and the result for $m=1$ follows again from an application of the maximum principle and the Hopf Lemma.

\section{Proof of Theorem 1.1}

We present now the proof of the long time existence theorem. The problem (2) is equivalent to a scalar graph evolution over the initial hypersurface, on a half space with an oblique derivative boundary condition. Notice here that one does not have a height bound for arbitrary scalar graphs evolving over a fixed half space in $\mathbb{R}^{n}$. Nevertheless we can apply the general parabolic theory for a graph over the initial surface to obtain a long time existence result as follows. We use results found in [11, Chapter 4] and obtain a unique solution for the associated linearised problem with smooth coefficients. The same fixed point arguments as for example in [12, Chapter 8], can then be applied to obtain a short time solution of the quasilinear scalar graph evolution in a half space with oblique derivative boundary condition. Following [11] we denote by $H^{1}$ the space of differentiable functions with continuous derivative and by $H^{1+\alpha}$ the space of differentiable functions with Hölder continuous derivative with exponent $\alpha \in(0,1)$.

If we have an initial height bound then for any time $t \geq 0$ we have $H^{1}$ uniform estimates on the solution, which together with the uniform estimate of the time derivative for the scalar graph function, given by the bound on the mean curvature, imply uniform $H^{1+\alpha}$ bounds (where $\alpha \in(0,1)$ ), as in [11, Chapter $6 \mathrm{Thm}$. 2.1]. The $H^{1+\alpha}$ uniform estimates for all times $t \geq 0$ imply long time existence by a standard application of the Arzelá-Ascoli theorem as in [12, Cor. 8.10].

If an initial height bound is not available we obtain long time existence regardless. The difference here is that the limit solution may not formally exist (as it does above). Suppose that the graphs over the initial hypersurface only exist for a finite time $T<\infty$. Since the gradient and the curvature estimates are uniform in time this implies that at time $T$ we have a smooth (at least $H^{1}$ ) hypersurface which allows us to reapply the short time existence theorem for a graph evolving over the hypersurface $M_{T}$ and contradict the maximality of $T$. Hence we have a solution of (2) for all time. 


\section{Asymptotic Behaviour}

In this Section we include the proof of the convergence result.

Proof of Theorem 1.2. If we have a height bound the result follows using Proposition 3.8 and taking $t \rightarrow \infty$ in

$$
H^{2} \leq \frac{n}{2 t}\left(\sup _{M_{0}} \frac{1}{s^{2}}-1\right) .
$$

This, together with (3) implies that $H \rightarrow 0$ as $t \rightarrow \infty$. Note that for this to be valid we require a formal limiting solution, as obtained above from the application of the Arzelá-Ascoli theorem. The same argument for the decay of second fundamental form tells us that the limit minimal surface is actually a hyperplane.

If we do not have an initial height bound, which is the case if we do not have the extra condition $s(F) \rightarrow 1$ as $|F| \rightarrow \infty$, then the result of Proposition 3.8 does not give us enough information to be able to state a convergence to hyperplane result. As noticed by Ecker-Huisken [4], the hypersurfaces can move out to infinity with speed proportional to $t^{-\frac{1}{2}}$. The global shape of the solution can be obtained for the rescaled flow (4) under the extra initial condition (5). The proof follows [4] with additional arguments to deal with the boundary.

The normalized equation for the rescaled flow (4) becomes

$$
\frac{d \tilde{F}}{d r}=-\tilde{H} \tilde{\nu}-\tilde{F}
$$

and one can easily see that the following estimates for the inverse gradient function $s$ and the second fundamental form hold for the rescaled flow

$$
\begin{aligned}
\tilde{s} & \leq C_{s}, \\
|\tilde{A}|^{2} & \leq C_{a},
\end{aligned}
$$

where $C_{s}$ and $C_{a}$ depend only on the initial surface $M_{0}$. The convergence result for the rescaled flow follows from the estimate

$$
\sup _{\tilde{M}_{r}} \frac{(\tilde{H}+\langle\tilde{F}, \tilde{\nu}\rangle)^{2}}{\tilde{s}^{2}\left(1+\alpha|\tilde{F}|^{2}\right)^{1-\epsilon}} \leq \exp ^{-\beta r} \sup _{M_{0}} \frac{\left(H+\left\langle F, \nu_{M_{0}}\right\rangle\right)^{2}}{s^{2}\left(1+\alpha|F|^{2}\right)^{1-\epsilon}},
$$

which we prove below holds for all $0<\epsilon<\delta$ and some constants $\alpha>0, \beta>0$ depending only on $\epsilon, n, C_{s}$ and $C_{a}$.

We now show that this estimate holds also in our boundary setting. The next lemma is the boundary analog of that found in [4].

Lemma 5.1. Let $\Sigma$ be a hyperplane in $\mathbb{R}^{n+1}$ with $n \geq 2,\left\langle\nu_{\Sigma}, e_{n+1}\right\rangle=0$ and $0 \in \Sigma$. Suppose $F_{t}$ satisfies $(2)$ and $F_{0}$ satisfies the initial graph condition (3) and condition (5). Then on $\tilde{M}_{r}$ we have the estimate

$$
\langle\tilde{F}, \tilde{\nu}\rangle^{2} \leq C(r)\left(1+|\tilde{F}|^{2}\right)^{1-\delta}
$$

for all $r>0$ with the constant $C(r)$ depending only on $r$ and $C_{a}$. 
Proof. Since the constant in the desired estimate is time dependent we can easily carry out the proof for the initial flow and it holds also for the rescaled one. Let $f=\left\langle F, \nu_{M_{t}}\right\rangle$ and consider the test function $\rho=\mu_{1}^{\delta-1}$ with $\mu_{1}=1+|F|^{2}+2 n t$. We derive the following parabolic evolution

$$
\left(\frac{d}{d t}-\Delta^{M_{t}}\right) f^{2} \rho \leq C\left(f^{2} \rho+1\right),
$$

where $C$ is just another constant depending on $C_{a}$. From now on we denote all constants depending on $C_{a}$ and $t$ by $C$. As stated in [4] this evolution implies for the boundaryless case that $f^{2} \rho$ grows at most exponentially in time.

We reconstruct this part of the proof since we need to treat the boundary behaviour also. Let $g=f^{2} \rho+1-e^{C t}$, and note that

$$
\left(\frac{d}{d t}-\Delta^{M_{t}}\right) g \leq C g
$$

Assume that $g$ is initially negative. Then by the maximum principle this is true for all times, modulo bad behaviour on the boundary. That is we need to exclude boundary maxima of $g=f^{2} \rho+1-e^{C t}$. Assume that there exists a boundary point $F(p, t) \in \partial M_{t} \subset \Sigma$ such that $g$ has a maximum. Choose an orthonormal frame $\left\{\tau_{i}\right\}_{i=1, n}$ of $T M_{t}$ such that $\left.\tau_{i}\right|_{\partial M_{t}} \in T \partial M_{t}$ for all $i=1, \ldots, n-1$ and $\left.\tau_{n}\right|_{\partial M_{t}}=\nu_{\Sigma}$ as in Lemma 3.2. Then from the evolution of $g \leq 0$ up to the time $t$ of the maximum, and the Hopf Lemma, we get the following sign on the directional derivative:

$$
0<\nabla_{\nu_{\Sigma}} g=2 f \rho \nabla_{\nu_{\Sigma}} f+f^{2} \nabla_{\nu_{\Sigma}} \rho
$$

We can compute the two derivatives separately as follows. First,

$$
\nabla_{\nu_{\Sigma}} f=\nabla_{\nu_{\Sigma}}\left\langle F, \nu_{M_{t}}\right\rangle=\left\langle\nu_{\Sigma}, \nu_{M_{t}}\right\rangle+\sum_{i=1}^{n-1} h_{i n}\left\langle F, \tau_{i}\right\rangle+h_{n n}\left\langle F, \nu_{\Sigma}\right\rangle=0
$$

where we have used the boundary condition $\left\langle\nu_{M_{t}}, \nu_{\Sigma}\right\rangle=0$, Proposition 3.3, and the fact that since $0 \in \Sigma$ we also have $\left\langle F, \nu_{\Sigma}\right\rangle=0$. The directional derivative of $\rho$ can be computed in the same way

$$
\begin{aligned}
\nabla_{\nu_{\Sigma}} \rho & =(\delta-1) \mu_{1}^{\delta-2} \nabla_{\nu_{\Sigma}} \mu_{1}=2(\delta-1) \mu_{1}^{\delta-2}\left\langle\nabla_{\nu_{\Sigma}} F, F\right\rangle \\
& =2(\delta-1) \mu_{1}^{\delta-2}\left\langle\nu_{\Sigma}, F\right\rangle=0,
\end{aligned}
$$

again by using $0 \in \Sigma$.

The last two computations imply $\nabla_{\nu_{\Sigma}} g=0$, which is a contradiction with the strict sign given by the Hopf Lemma. Therefore there is no maximum of $g$ on the boundary and our proof is complete.

The following lemma can be found in [4].

Lemma 5.2 (Ecker-Huisken [4]). Let $F_{t}$ be a solution of mean curvature flow. Then the normalized quantity $\tilde{H}+\langle\tilde{F}, \tilde{\nu}\rangle$ satisfies the evolution equation

$$
\left(\frac{d}{d r}-\Delta^{\tilde{M}_{r}}\right)(\tilde{H}+\langle\tilde{F}, \tilde{\nu}\rangle)=\left(|\tilde{A}|^{2}-1\right)(\tilde{H}+\langle\tilde{F}, \tilde{\nu}\rangle) .
$$

We are now ready to prove inequality (17). 
Proof of inequality (17). Again we follow [4] setting $f^{2}=\frac{1}{s^{2}}(\tilde{H}+\langle\tilde{F}, \tilde{\nu}\rangle)^{2}$ and $\rho=$ $\mu_{\alpha}^{\epsilon-1} e^{\beta r}$ where $\mu_{\alpha}=1+\alpha|\tilde{F}|^{2}$, with $0<\epsilon<\delta$ and $\alpha, \beta$ are small positive constants to be determined. As in [4] one can use Lemma 5.2 and choose the constants such that $g=f^{2} \rho$ satisfies the following evolution on the rescaled hypersurface:

$$
\left(\frac{d}{d r}-\Delta^{\tilde{M}_{r}}\right) g \leq a \cdot \nabla^{\tilde{M}_{r}} g
$$

for $a$ a bounded vector field on $\tilde{M}_{r}$.

Lemma 5.1 ensures that $g$ vanishes at infinity which enables us to apply the maximum principle to conclude that, if the boundary behaviour is controlled, $g$ is uniformly bounded by its initial data.

On the boundary we are able to exclude maxima of $g$ by the same usage of the Hopf Lemma as in Lemma 5.1 and since the scaling is only in time we can also compute the directional derivative of $g$ on the unscaled manifold. That is,

$$
0<\nabla_{\nu_{\Sigma}} g=2 f \rho \nabla_{\nu_{\Sigma}} f+f^{2} \rho \nabla_{\nu_{\Sigma}} \rho
$$

We can compute the two directional derivatives separately as

$$
\begin{aligned}
\nabla_{\nu_{\Sigma}} f & =\frac{1}{s} \nabla_{\nu_{\Sigma}} H+\frac{1}{s} \nabla_{\nu_{\Sigma}}\left\langle F, \nu_{M_{t}}\right\rangle \\
& =\frac{1}{s}\left\langle\nu_{\Sigma}, \nu_{M_{t}}\right\rangle+\frac{1}{s} \sum_{i=1}^{n-1} h_{i n}\left\langle F, \tau_{i}\right\rangle+\frac{1}{s} h_{n n}\left\langle F, \nu_{\Sigma}\right\rangle=0,
\end{aligned}
$$

where we have used $\nabla_{\nu_{\Sigma}} s=0$ as in Proposition 3.6, the boundary condition $\left\langle\nu_{M_{t}}, \nu_{\Sigma}\right\rangle=0$, Proposition 3.3 for $\alpha \equiv 0$, the choice of frame and the fact that since $0 \in \Sigma$ then we also have $\left\langle F, \nu_{\Sigma}\right\rangle=0$. For the directional derivative of $\rho$ we may ignore only dependent upon time exponential and compute

$$
\nabla_{\nu_{\Sigma}} \mu_{\alpha}=2 \alpha\left\langle\nabla_{\nu_{\Sigma}} F, F\right\rangle=2 \alpha\left\langle\nu_{\Sigma}, F\right\rangle=0,
$$

again using $0 \in \Sigma$. The last two equations contradict (18) and thus there is no boundary maximum for $g$. This completes the proof of Theorem 1.2.

As in [4] we are able to prove that the only contracting self similar solutions of (2) are hyperplanes. The essential point is that the boundary integrals resulting from integration by parts all vanish.

Proposition 5.3. Let $\Sigma$ be a hyperplane in $\mathbb{R}^{n+1}$ with $n \geq 2,\left\langle\nu_{\Sigma}, e_{n+1}\right\rangle=0$. If $M$ is a solution of (2) satisfying (3) and the equation

$$
H=\left\langle F, \nu_{M}\right\rangle \text {, }
$$

then $M$ is a hyperplane.

Proof. From (19) we can compute $\nabla_{i} H=\left\langle F, \tau_{j}\right\rangle h_{i j}$ with a choice of orthonormal frame as in Lemma 3.2. Using the evolution of $s$ we have

$$
\Delta^{M} s=-|A|^{2} s+\sum_{i=1}^{n} \nabla_{i} s\left\langle F, \tau_{i}\right\rangle .
$$

Noting that in a choice of orthonormal frame such that $\left.\tau_{n}\right|_{\partial M}=\nu_{\Sigma}$ we have $\nabla_{\nu_{\Sigma}} s=$ 0 ( cf. Proposition 3.6) we multiply this equation by the test function $\rho=e^{-\frac{|F|^{2}}{2}}$ 
and integrate by parts to obtain

$$
\int_{M}|A|^{2} \operatorname{s\rho d} \mu=0
$$

which implies the result.

\section{Optimality of CONVERGence}

In this section we show that the result of Theorem 1.2 is optimal. That is, for an initial surface which slowly oscillates at infinity, violating condition (5), one does not expect asymptotic convergence as described in the above section. This is an adaptation of $[4$, Section 6$]$ to our situation.

Lemma 6.1. Suppose that $M_{0}$ and $F_{t}$ satisfy the assumptions of Proposition 3.9 and suppose there exists a sequence of points $p_{k} \in M^{n}$ such that $\left|F\left(p_{k}, 0\right)\right| \rightarrow \infty$ and $\left\langle F\left(p_{k}, 0\right), \nu_{M_{0}}\right\rangle^{2}=\gamma\left|F\left(p_{k}, 0\right)\right|^{2}$ for some $\gamma>0$. Then there exists a sequence of times $r_{k}$ for which $(\tilde{H}+\langle\tilde{F}, \tilde{\nu}\rangle)$ does not converge to zero.

The proof does not require any boundary estimates and it is thus identical to the one found in [4]. It is based on the results of spatial decay of curvature obtained in Proposition 3.9.

\section{ACKNOWLEDGEMENTS}

The results from this paper are partially contained in the author's Ph.D. thesis [14], under the supervision of Prof. Dr. Klaus Ecker at the Freie Universität in Berlin, in the group of Geometric Analysis as a Berlin Mathematical School student. The author would like to thank them for financial support and help during the completion of this paper and her thesis.

\section{REFERENCES}

[1] S.J. Altschuler and L.F. Wu. Translating surfaces of the non-parametric mean curvature flow with prescribed contact angle. Calc. Var. Partial Differential Equations, 2:101-111, 1994.

[2] J.A. Buckland. Mean curvature flow with free boundary on smooth hypersurfaces. J. Reine Angew. Math., 586:71-90, 2005.

[3] K. Ecker. Regularity Theory for Mean Curvature Flow. Birkhauser, 2004.

[4] K. Ecker and G. Huisken. Mean curvature evolution of entire graphs. Ann. of Math., 130(2):453-471, 1989.

[5] K. Ecker and G. Huisken. Interior estimates for hypersurfaces moving by mean curvature. Invent. Math., 105(1):547-569, 1991.

[6] B. Guan. Mean curvature motion of nonparametric hypersurfaces with contact angle condition. Elliptic and parabolic methods in geometry, pages 47-56, 1996.

[7] G. Huisken. Flow by mean curvature of convex surfaces into spheres. J. Differential Geom., 20(1):237-266, 1984.

[8] G. Huisken. Contracting convex hypersurfaces in Riemannian manifolds by their mean curvature. Invent. Math., 84(3):463-480, 1986.

[9] G. Huisken. Non-parametric mean curvature evolution with boundary conditions. J. Differential Equations, 77:369-378, 1989.

[10] A. Koeller. On the Singularity Sets of Minimal Surfaces and a Mean Curvature Flow. PhD thesis, Freie Universität Berlin, 2007.

[11] O.A. Ladyshenzkaya, V.A. Solonnikov, and N.N. Uralceva. Linear and quasilinear equations of parabolic type, Translations of Mathematical Monographs, Band 23. Amer. Math. Soc., Rhode Island, 1968.

[12] G.M. Lieberman. Second order parabolic differential equations. World Scientific Pub. Co. Inc., 1996. 
[13] A. Stahl. Über den mittleren Krümmungsfluss mit Neumannrandwerten auf glatten Hyperflächen. PhD thesis, Fachbereich Mathematik, Eberhard-Karls-Universität, Tüebingen, Germany, 1994.

[14] V.M. Vulcanov. Mean curvature flow of graphs with free boundaries. PhD thesis, Freie Universität, Fachbereich Mathematik und Informatik, Berlin, Germany, 2010.

Valentina Mira Wheeler, Fachbereich Mathematik und Informatik, Freie UniverSität, Arnimallee 3, 14195 Berlin Germany, email: vulcanov@zedat.fu-Berlin.de 